\title{
A case report of hidradenitis suppurativa after subdermal excision for axillary osmidrosis
}

Joon Shik Hong, , Jung Hwan Kim ${ }^{1}$, Gyu Yong Jung ${ }^{1}$, Joon Ho Lee ${ }^{1}$, Tae Jung Jang ${ }^{2}$, Hea Kyeong Shin ${ }^{1}$

Departments of ${ }^{1}$ Plastic and Reconstructive Surgery and ${ }^{2}$ Pathology, Dongguk University College of Medicine, Gyeongju, Korea
Hidradenitis suppurativa (HS) is a chronic inflammatory condition with an unclear etiopathogenesis that is considered to be a follicular occlusive disease. We present a case of $\mathrm{HS}$ that was suspected to have developed as a complication of subdermal excision. A 19-year-old man who had undergone subdermal excision due to osmidrosis presented 7 months after surgery with a persistent painful mass in his left axilla. Despite medical treatment, incision, and drainage, a painful enlarged abscess recurred in the left axilla and was cured completely by deroofing surgery. However, 15 months after subdermal excision, he revisited the hospital because of a painful mass in the right axilla. The patient's condition met the diagnostic criteria of HS. After several recurrences, a cure was achieved by radical wide excision. Mechanical stress like that associated with subdermal excision is considered to be a possible etiological factor of HS. In addition, pathological changes at the sebofollicular junction allow rupture and leakage of folliculopilosebaceous units upon exposure to mechanical stress, which may result in the aggressive subcutaneous extension of inflammation. We suggest that HS should be considered in patients with a recurrent abscess after subdermal excision, and recommend surgical treatment as a possible option if conservative treatment is clinically ineffective.

Keywords Hidradenitis suppurativa / Apocrine glands / Mechanical stress / Friction / Odorant

\section{INTRODUCTION}

Hidradenitis suppurativa (HS) is a chronic, inflammatory, follicular occlusive skin disease that is characterized by recurrent, painful, deep-seated nodules and abscesses in so-called inverse areas, such as the axillary, inguinal, and genital regions [1]. All these regions are apocrine gland-bearing areas; therefore, HS was initially considered to be an inflammatory process associated with apocrine

Received: Dec 12, 2019 Revised: Jan 19, 2020 Accepted: Jan 28, 2020

Correspondence: Hea Kyeong Shin Department of Plastic and

Reconstructive Surgery, Dongguk University College of Medicine,

87 Dongdae-ro, Gyeongju 38067, Korea

Tel: +82-54-770-1460, Fax: +82-54-770-1469,

E-mail: shinheakyeong@hanmail.net

Copyright @ 2020 The Korean Society for Aesthetic Plastic Surgery.

This is an Open Access article distributed under the terms of the Creative Commons Attribution Non-Commercial License (https://creativecommons.org/licenses/by-nc/4.0/) which permits unrestricted non-commercial use, distribution, and reproduction in any medium, provided the original work is properly cited. $\quad w w w . e-a a p s . o r g$ sweat glands. However, since the 1990s, inflammation of the apocrine sweat glands has been considered to be a secondary change resulting from follicular occlusion. The cause of HS is unclear and multifactorial, although identified etiological factors include genetic susceptibility, obesity, smoking, and hormonal factors [2]. Furthermore, mechanical stresses and shear forces are considered to play possible etiological roles [3].

Here, we present a case of HS that we suspect developed as a complication of subdermal excision of apocrine glands, and include a review of the literature. In addition, we speculate on the sequence of events that led to HS in this case.

\section{CASE REPORT}

A 19-year-old male smoker with a normal body mass index (23.88 $\mathrm{kg} / \mathrm{m}^{2}$ ) underwent bilateral subdermal excision due to osmidrosis at our hospital in December 2014. The surgical procedure was as follows. Two skin incisions were made parallel to the axillary creases. Defatting of the flap and removal of the apocrine glands were 
performed after subdermal undermining. The incision was closed with Nylon 5-0 sutures and a tie-over dressing was placed on each axilla.

The patient had no previous history of HS lesions in inverse skin areas or a family history of HS. The patient, who had grade 3 malodor, was satisfied with the relief of that symptom postoperatively [4]. However, after 7 months, he presented at our hospital because of intermittent pain associated with a scar in his left axilla, which

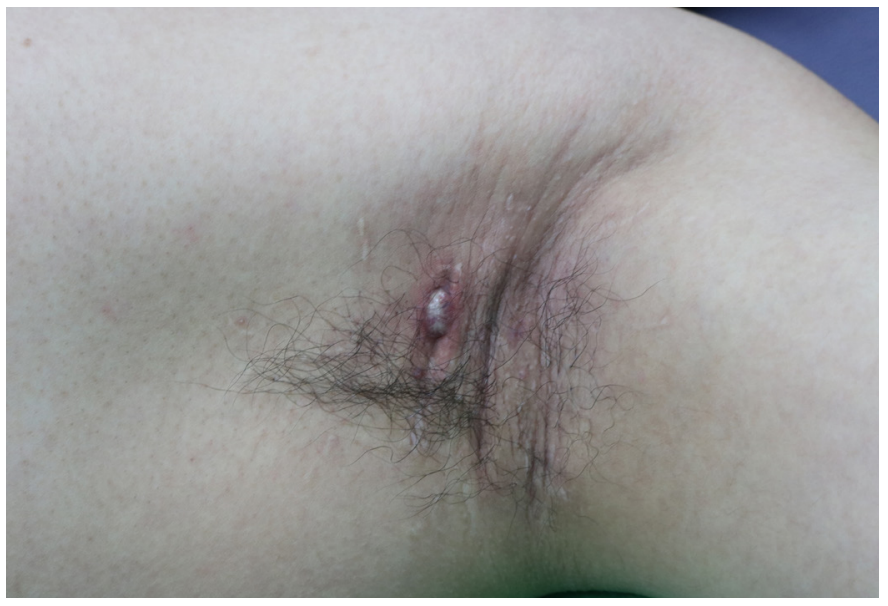

Fig. 1. Thirteen months after subdermal excision was performed for osmidrosis. A $1.5 \times 1 \mathrm{~cm}$ painful abscess with surrounding scar tissue developed in the left axilla. It was located approximately $1.0 \mathrm{~cm}$ away from the first surgical incision.

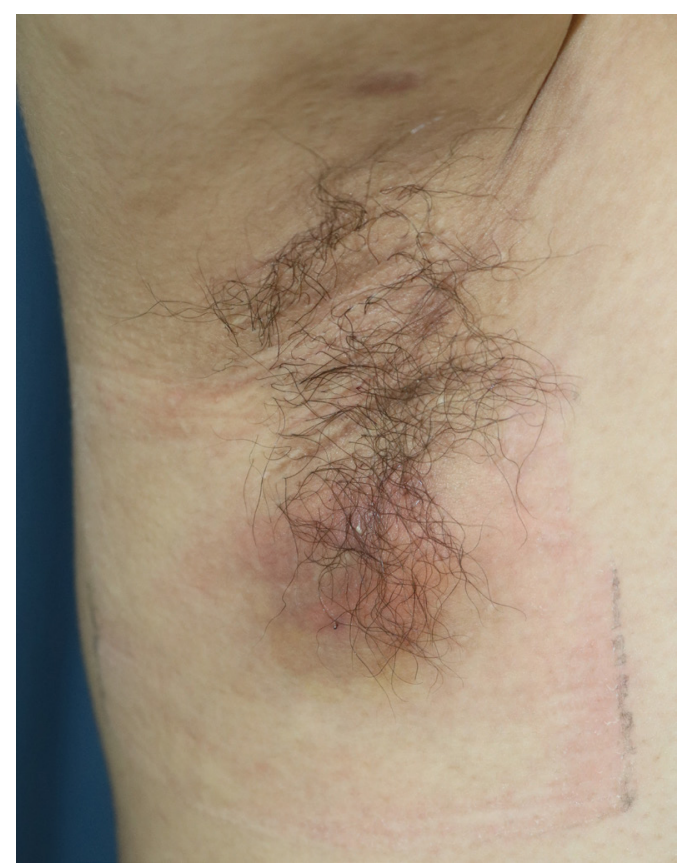

Fig. 2. Fifteen months after subdermal excision was performed for osmidrosis. A $4 \times 4 \mathrm{~cm}$ abscess had developed in the right axilla. was treated by a triamcinolone injection and cephalosporin antibiotics. Although this medical treatment, along with incision and drainage, helped to relieve his symptoms temporarily, he returned 13 months after the initial surgery with abscesses in the left axilla, including one that measured $1.5 \times 1 \mathrm{~cm}$ (Hurley stage I) with redness and scarring of the surrounding tissue (Fig. 1). The abscesses were surgically removed using the deroofing technique, and after that procedure, inflammatory lesions of the left axilla did not recur.

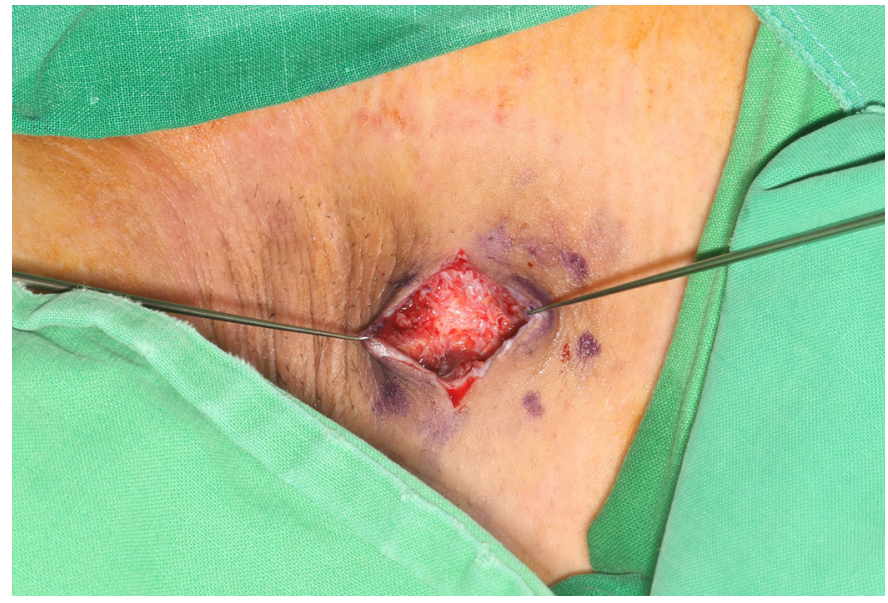

Fig. 3. Intraoperative photograph of limited local excision. Intraoperatively, an irregularly indurated cystic lesion was observed with surrounding fibrotic scar tissue.

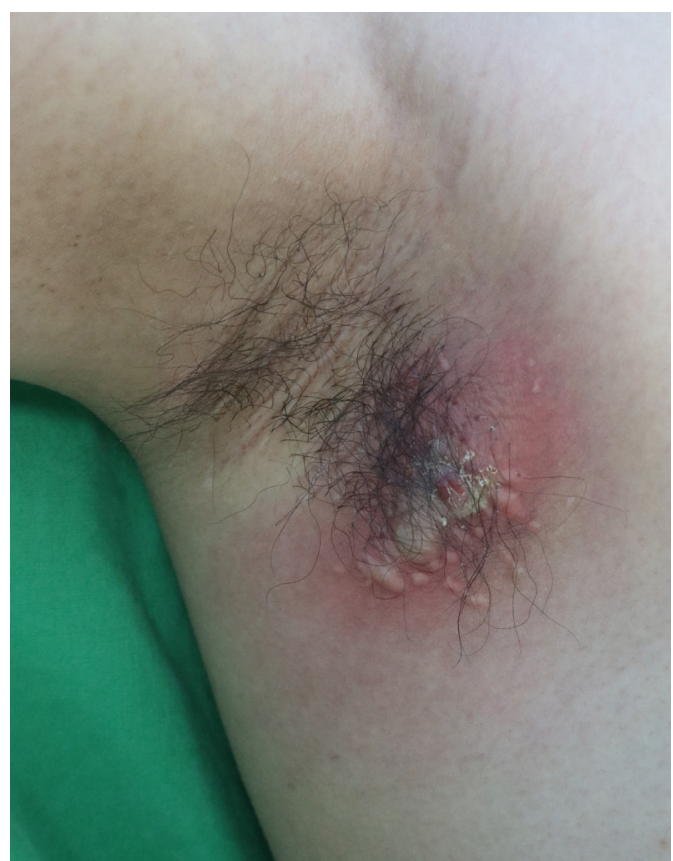

Fig. 4. Three years after subdermal excision was performed for osmidrosis. An enlarged deep-seated painful abscess $(7 \times 4 \mathrm{~cm})$ had developed in the right axilla. 

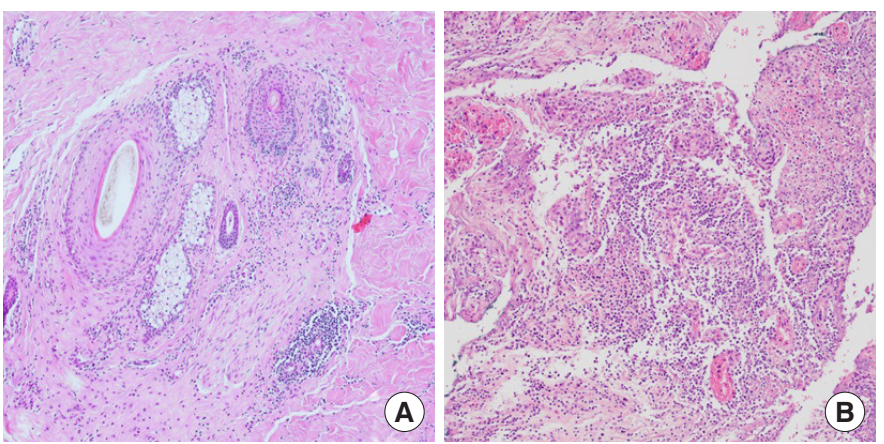

Fig. 5. Tissue sections show chronic inflammation around and within the hair follicles $(A)$, with abscess formation in the deep dermis (B) (H\&E, $\times 100)$.

Nonetheless, in March 2016 (15 months after subdermal excision), the patient presented again with swelling and pain in the right axilla (Fig. 2). Upon physical examination, a soft, subcutaneous, $4 \times 4 \mathrm{~cm}$ abscess with unclear margins was palpated. Broad redness and swelling were also observed around the abscess and were accompanied by severe tenderness. We removed the inflamed cystic lesion and surrounding fibrotic tissues (Fig. 3). Antibiotics and anti-inflammatory drugs were administered after excision of the lesion, after which the inflammation began to subside and symptoms disappeared. However, 3 months after excision of the lesion, severe HS of the right axilla recurred (Hurley stage II). A physical examination showed extensive redness and pain in a region of $7 \times 4 \mathrm{~cm}$ around the previous site (Fig. 4). Radical wide excision was performed and the specimen was sent to the pathology department for biopsy (Fig. 5). The skin defect caused by the wide excision was repaired by primary repair. This was possible because the skin around it had sufficient flexibility. Additionally, antibiotics and anti-inflammatory drugs were administered, and as of the most recent followup (3 months after the last operation), there had been no recurrence (Fig. 6).

\section{DISCUSSION}

HS is diagnosed based on a history of recurrent painful suppurative abscesses, in inverse areas, with scarring of the sinus tracts. Its diagnostic criteria were adopted by the Second International HS Research Symposium, as described below [1]: (1) typical lesions (deep-seated painful nodules or abscesses, draining sinuses, bridged scars); (2) typical locations (axilla, groin, genitals, perineum, perianal region, buttocks, and inframammary folds); and (3) chronicity and recurrence.

The most common differential diagnoses are the follicular pyodermas (folliculitis, furuncles, and carbuncles). In the case described herein, the patient had suffered from recurrent pain and abscess formation in both axillae over a period of 19 months. When wound culture was performed on the right axilla to exclude other infec-

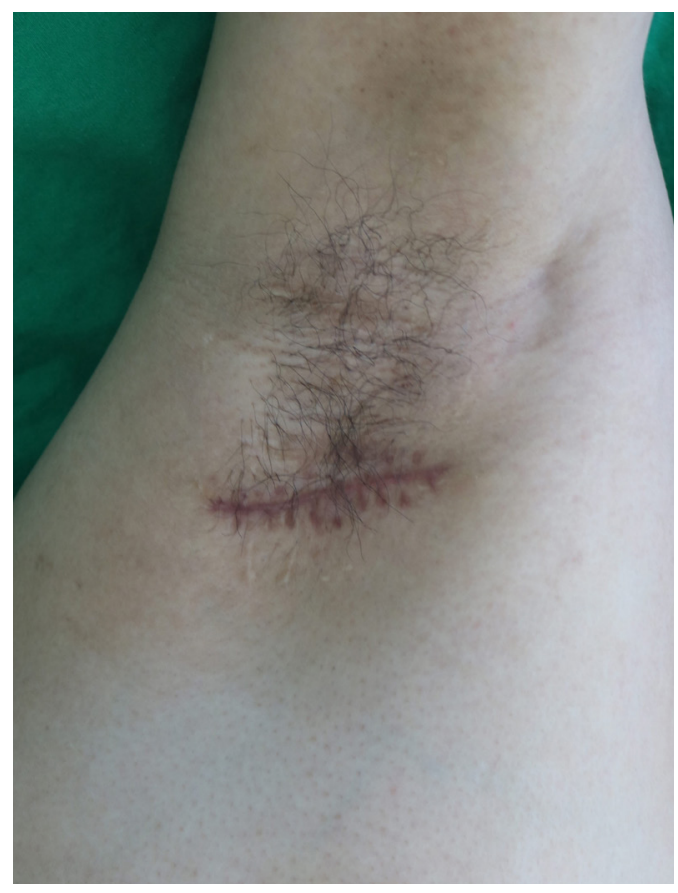

Fig. 6. Six months after radial wide excision. The inflammation had completely disappeared and there was no evidence of recurrence.

tious diseases [5], no bacteria of any kind were detected in the lesion, enabling other infectious diseases to be excluded. In addition, the pathological findings also supported the diagnosis of HS (Fig. 5).

In 1989, Hurley proposed a classification system for HS based on three disease severity stages. Stage 1 is characterized by single or multiple abscesses without cicatrization or a sinus tract, stage 2 by one or more widely separated recurrent abscesses with tract formation, and stage 3 by multiple interconnected tracts and abscesses throughout an entire area [5,6]. In our case, the patient had recurrent multiple abscesses with scar formation in the right axilla, and thus, was classified as Hurley stage 2 .

The treatment of HS can be classified as medical or surgical. Medical treatments are based on antibiotics, antiandrogens, and immunosuppressants. However, since no single, uniformly effective therapy is available for HS, surgical management should be performed if medical treatment fails. Surgical management strategies include incision and drainage, deroofing and marsupialization, limited local excision, and radical wide excision. Defect resurfacing after radical wide excision is important for maintaining function, reducing contracture, and obtaining a good aesthetic outcome. The options for reconstruction are primary closure, healing by secondary intention, split-thickness skin grafting (immediate or delayed), and fasciocutaneous or myocutaneous flaps. When the disease is extensive, it is necessary to perform wider excision, which requires complete removal of all folliculopilosebaceous units in the affected region $[7,8]$. In our patient, we treated the right axillary lesion by radi- 
cal wide excision when it recurred after limited local excision.

Identified etiological factors of HS include genetic susceptibility, obesity, smoking, and hormonal factors [2]. In particular, genetic susceptibility appears to play an important role in HS. Patients with HS have a $35 \%$ to $40 \%$ positive family history, with an inheritance pattern consistent with autosomal dominant inheritance. Overall, $43 \%$ to $77 \%$ of patients with HS are overweight or clinically obese, which may worsen or possibly trigger HS due to the frictional effects of skin folds. Furthermore, $70 \%$ to $89 \%$ of patients with HS have a history of smoking, and nicotine has been shown to activate nonneuronal acetylcholine receptors, causing increased keratinization of the pilosebaceous duct, which suggests that nicotine may play a role in the development of HS. When hormonal overstimulation of the production of ductal keratinocytes results in failure of terminal differentiation of the keratinocytes lining the ducts, keratinocytes accumulate in a structure known as a comedo. Of these possible etiological factors, smoking was the only relevant factor in our patient.

Several case reports support the notion that mechanical stress contributes to the development of HS [3,9-11], and it has also been suggested that defective follicular support is an etiological factor. Interestingly, the sebofollicular junction in HS patients has been reported to exhibit thinning of periodic acid-Schiff-positive material along the basement membrane, which may explain the apparent fragility of the sebofollicular junctions of the folliculopilosebaceous units [12], and why individuals with such a predisposition tend to contract HS when exposed to mechanical stress.

We postulate that the following sequence of events occurred in our patient. (1) A primary pathogenic change occurred in the sebofollicular portions of the folliculopilosebaceous units. (2) Mechanical stress caused by direct injury to the folliculopilosebaceous units-either friction or pressure induced by subdermal excision, or resulting from the tie-over dressing-provoked rupture or leakage of the folliculopilosebaceous units. (3) The release of keratin fragments generated inflammatory stimuli. (4) The innate and adaptive immune systems were activated. (5) Immune cells infiltrated the surrounding dermis, leading to chronic inflammation and formation of an invasive proliferative gelatinous mass, (6) resulting in the formation of horizontal sinuses and scarring (Fig. 7).

To summarize, no case of HS after subdermal excision for axillary osmidrosis has been previously reported. As a result of our experiences with this case, we suggest that HS should be considered when a recurrent painful mass without obvious signs of infection is encountered after subdermal excision for osmidrosis. Furthermore, we recommend wide radical excision if medical treatment or incision and drainage alone do not have any clinical effect. Finally, we emphasize that care must be taken to adequately treat HS because it is known to be associated with elevated risks for squamous cell carcinoma and other malignancies [5].
Weakness in the support structure of the sebofollicular junction of the FPSU

Rupture and leakage of the FPSU by mechanical stress

Release of keratin fragments and inflammatory stimuli

Trigger innate and adaptive immune systems

Formation of an invasive proliferative gelatinous mass

Scarring process of HS

Fig. 7. Proposed pathogenic model and sequence of events. FPSU, folliculopilosebaceous units; HS, hidradenitis suppurativa.

\section{NOTES}

\section{Conflict of interest}

No potential conflict of interest relevant to this article was reported.

\section{Ethical approval}

The study was approved by the Institutional Review Board of Dongguk University Gyeongju Hospital (IRB No. 110757-201910-HR02-02) and performed in accordance with the principles of the Declaration of Helsinki.

\section{Patient consent}

The patient provided written informed consent for the publication and the use of his images.

\section{ORCID}

Joon Shik Hong

Jung Hwan Kim

Gyu Yong Jung

Joon Ho Lee

Tae Jung Jang

Hea Kyeong Shin

\section{REFERENCES}

1. Diagnostic Criteria. Second International HS Research Symposium 2009. San Francisco: Hidradenitis Suppurativa Foundation, Inc.; 2009.

2. Danby FW, Margesson LJ. Hidradenitis suppurativa. Dermatol Clin 2010;28:779-93.

3. de Winter K, van der Zee HH, Prens EP. Is mechanical stress an im- 
portant pathogenic factor in hidradenitis suppurativa? Exp Dermatol 2012;21:176-7.

4. Park YJ, Shin MS. What is the best method for treating osmidrosis? Ann Plast Surg 2001;47:303-9.

5. Alikhan A, Lynch PJ, Eisen DB. Hidradenitis suppurativa: a comprehensive review. J Am Acad Dermatol 2009;60:539-61.

6. Napolitano M, Megna M, Timoshchuk EA, et al. Hidradenitis suppurativa: from pathogenesis to diagnosis and treatment. Clin Cosmet Investig Dermatol 2017;10:105-15.

7. Ingram JR. Hidradenitis suppurativa: an update. Clin Med (Lond) 2016; 16:70-3.

8. Wieczorek M, Walecka I. Hidradenitis suppurativa: known and unknown disease. Reumatologia 2018;56:337-9.
9. Rondags A, Diercks GF, Werker PMN, et al. Ectopic hidradenitis suppurativa on the dorsal foot of a road maker. JAAD Case Rep 2017;3: 429-31.

10. De Vita V, Fabbrocini G. Mechanical stress as a cause of hidradenitis suppurativa: a lesson from a patient with a monster hernia. Acta Dermatovenerol Croat 2018;26:260-1.

11. Boer J. Should hidradenitis suppurativa be included in dermatoses showing Koebnerization? Is it friction or fiction? Dermatology 2017; 233:47-52.

12. van der Zee HH, Prens EP. Preliminary findings suggest hidradenitis suppurativa may be due to defective follicular support. Br J Dermatol 2013;168:926-7. 